\title{
Research on the Development Status and Countermeasures of
}

\section{Community Taijiquan}

\author{
Guo Zhendong ${ }^{1}$ \\ ${ }^{1}$ Physical Culture Institute of Yan'an University, Yan'an, Shanxi Province, 716000
}

Keywords: Community members, Taijiquan, Status analysis, Development strategies

\begin{abstract}
The community sport is an effective way to carry out extensive mass fitness programs and it attracts many community members with its flexible organization forms, harmonious local atmosphere, easy practicing methods and sound effects. This paper makes an overall exploration on the Tanjiquan organization and its development status in some communities in Yan'an with questionnaires, literature, interview and mathematical statistics methods. The author analyzes the organization, management and development problems of community Taijiquan and proposes the development countermeasure.
\end{abstract}

\section{Introduction}

Taijiquan is a martial art of boxing with a long history. It has high fitness value and unique charm of oriental culture with the opening and closing of yin and yang, the conversion between deficiency and excess, the combination of hardness and softness and the inter-phase with speed and slowness. With the deepening understanding of community construction, the community sport has become the people's focus with its closeness to people's lives. With the extensive development of community activities and people' increase needs of community fitness and recreation, Taiqiquan with its fitness, self-defense, self-cultivation, entertainment and other functions adapts to and meet the modern demands of people with different levels and services the human health business. Therefore, in order to adapt to the development of mass sports and improve athletic level of Taijiquan in Yan'an, this article makes an investigation and research on its development status, problems and trends.

\section{Research Objects and Methods}

Research objects. People in three squares, three parks and 21 communities in urban area of Yan'an.

Research methods. (1) Questionnaire method. The author distributed 300 questionnaires to people in three squares, three parks and 21 communities in urban area of Yan'an and took back 268 questionnaires with $89 \%$ recovery rate. The valid questionnaires are 259 , accounting for $97 \%$ of returned questionnaires. (2) Interview method. The author interviewed persons in charge of communities in Yan'an. (3) Mathematical statistics and theoretical analysis method. The author took mathematical statistics and theoretical analysis of the obtained information.

\section{The Analysis of the Development Status}

The activity scale. According to the survey, the current size of Taijiquan in Shanxi Province is generally small and the number of exercise people at different points is inconsistent (Table 1). As you can see from Table 1, in community sports points, groups with 1-9 people accounts for $21 \%$, 
groups with10-30 people represents 52\%; groups with 30-50 accounts for about 22\%; groups with more than 50 people is $5 \%$. By this token, the activity scale is small and these smaller points are equivalent of a primary social group. People are connected by a close living relation, which is closely related to China's cultural tradition. Traditional agricultural society creates relation networks with a limited number of people. They have close relation and combine themselves with a sense of friendship and responsibility. It is convenient for few people to share beliefs and values with other members. You can see from the survey that groups with 10-30 people share relatively a large proportion.

Table 1 The scale of community members participating in Taijiquan (\%)

The number of people

Percent (\%)

\section{$1-9$}

$10-20$

20-30

$\geq 50$

\section{1}

52

22

5

Members at activity points. In term of gender, participants at each community sport point in Yan'an have common features. The proportion of women is larger than men and the proportion of older people is greater than the young. In the returned questionnaires, $68 \%$ are women and men accounts for only 32\%, while the proportion of the elderly shares $61 \%$ and only $39 \%$ are young people. In term of people's occupation, the reason why the elderly shares the largest proportion is that they are retired at home and have more leisure time, while the reason that the number of re-employed young people is fewer is mainly their restrictions on working time. Among the elderly, persons aged 40-50 account for only $11 \%$ of the number of the elderly, 50-70 year-old people shares $72 \%$ and people aged 70 or older accounts for $17 \%$. In terms of gender, women are more than man.

Members' exercise purposes. The members' main purposes for Taijiquan are the health improvement, diseases prevention, athletic rehabilitation, emotional adjustment, pressure remission, diet fitness, entertainment and social networking in sequence. It can be seen that most of the elderly take Taijiquan exercise to cure or prevent diseases, while the motives of young people are to ease pressure caused by work or study, to adjust emotions, to lose weight and to entertain. Moreover, Taijiquan has become a means to conduct athletic rehabilitation and increase communication between residents, which generates more development approaches to promote Taijiquan.

Members' exercise time and frequency. The Taijiquan activity time in Yan'an is relatively fixed and people with different ages have different activity time. Survey results show that $82 \%$ of activity people have a fixed time and only $18 \%$ do not have a fixed time. Because the elderly have more leisure time, their activity time is long, generally more than one hour. Young people activity time usually lasts 30-50 minutes due to work or study. However, there is a common feature between these two groups that is they are accustomed to exercise in the morning. With age growing, old people's sleep time is reducing and their life habits will be more regular. They will get up and sleep early, so the morning becomes their preferred exercise time. While young people's life habits are relatively irregular and they have many evening activities. The morning time is often their only exercise time. From this perspective, we can see that Taijiquan exercise time in Yan'an is mainly the morning. In terms of exercise frequency, exercise frequency is different for different people and 
they want to do exercise based on their own time. Survey results show that people who do excises more than three times a week accounts for $73 \%$ of the total population; people who do excises $1-2$ times a week shares $21 \%$. People who have no fixed excises time in the survey accounts for $6 \%$. 93\% people who do excises three times a week will do exercise every day. With the further implementation of five-day work and paid leave, it is foreseeable that people's time to participate in Taijiquan will be more flexible.

Activity places. Members with different exercise time have various activity places and they will choose excise places based on their dwelling environment. The survey shows that activity places are usually following by green squares, space in the community, parks, free gymnasiums, nearby streets and charged gymnasiums. Most of the people choose free exercise places. Though the government has built many sports centers and the green squares in recent years, the gymnasiums still seem relatively scarce.

Members' Consumption. In terms of Taijiquan exercisers' consumption, people's consumption includes the purchase of clothes, equipment, books with related knowledge. However, the number of people who are willing to pay to attend a tutorial class accounts for only $22 \%$. It can be seen that people have not cultivated sport consumption awareness and they do not have the spending-money-on-health concept. People, who are not yet accustomed to the market economy, still lay the exercise task on the body of the country and want the state to spend more money to maintain sports excises.

The Management of activity places. From the view of internal management of the community Taijiquan, most places are inattentive and members have free access to them. There is little formal management, and no organization rules and regulations. However, with the development of activity places, a number of activity places form initial organizations. For example, a few people start to manage places and convene people. There is a more clear division of labor and the internal management is initially formed. According to the survey, standardized organizations account for only $4 \%$; people with organizations share $49 \%$ of all people, while the loose organizations accounts for $47 \%$. Taijiquan activities are usually organized temporarily in many communities and the spontaneity is strong. From the view of the relation between activity places and the external, many activity places have no contact with the external. Most people take exercise spontaneously and they attract each other. In terms of activity instructors, in our survey, half of the activity places have no instructors and some instructors who direct members are the convener. There are only eight places with professional instructors, but most of them have lower degrees and the professional level is not high. Although the country has implemented social sports instructor system and has cultivated a considerable number of instructors so far, there is a wide gap between public's growing demand for fitness and recreation and instructors' level.

\section{The Development Countermeasure of Community Taijiquan}

To strengthen the systematization of community Taijiquan sports organizations. Community Taijiquan organizations in Yan'an basically form a multi-level system, but the organization form is still loose and mainly developed by small spontaneous ones. According to the survey, more than $65.6 \%$ of the residents are willing to pay lower fee to participate in standard physical activities in sports organizations with professional instructors. The commercial fitness organizations have higher charges, which will not become the mainstream of popular sports. Therefore, in order to meet the needs of Taijiquan development and promote the further development of Taijiquan, we must strengthen the construction of Taijiquan organizations. We can develop public welfare folk Taijiquan organizations and their basic features are following: it is civil, which is non-governmental; 
the main purpose of these activities is to work for the public good and service society, which undertake activities with non-profit purposes. Therefore, with the current situation of our country, in order to strive to foster the development of civil Taijiquan organizations, on one hand, we should diversify social organizations to enhance the diversification of economic market; on the other hand, we should promote the reform of government and make some civil organizations charge of some social management and social service functions which are controlled by government previously.

To enhance the management network of community Taijiquan organizations. The management network of community Taijiquan organizations is the inevitable trend for community sports management to fit development of modern society. With the growth of the desire for physical fitness and multi-level needs, only to further strengthen the management network of community Taijiquan organizations, can we meet people's need for Taijiquan and can we systematically improve urban community sports work. As a basic link in the network of community sports organizations, urban sub-district offices are in charge with the historical responsibility to connect the preceding and the following. To strengthen management functions of the sub-district offices in the urban community sports work is the key to successfully carry out the work of urban community Taijiquan.

To promote the amateurish development of community Taijiquan. Tanjiquan activities carried out by athletic associations in sub-district communities and regional athletic associations in Yan'an have not been amateurish. With the economic development, the reduction of public institution members and the accelerating pace of work, professional sports will be more restricted. Morning, evening and two-day weekend will be fully utilized and the amateurish degree of Taijiquan activity time will continue to increase.

To intensify propaganda for Taijiquan knowledge and enhance residents' fitness concepts. We need to give full play to the mass media to direct community Taijiquan and to further promote community awareness of all age groups. Furthermore, we can use the blackboard newspapers and bulletin boards to provide fitness knowledge and we may distribute some popular and instructive fitness books that are suitable for readers with different levels. Moreover, we should enhance information campaigns for Taijiquan features, eliminate wrong workout ideas and guide people to implement the right way to exercise, so that people can actively and consciously participate in the workout.

To strengthen the guidance and organization management of community Taijiquan. Community Taijiquan in Yan'an requires both a certain degree of organization and management and a number of Taijiquan instructors. Some universities, such as physical culture institute in Yan'an Normal University, have the advantage of personnel with strong guidance and management and we can send excellent students with theoretical and comprehensive knowledge and high technical level to guide and organize community fitness activities in weekends or after school. In addition, the government can use its functions and send specialized personnel to instruct community members at a certain time. This will improve their professional knowledge and skills.

\section{References}

[1] Li Peng. A Research on the Status and Countermeasures of Community Taijiquan in Guangzhou [J]. Journal of Physical Education, 2001, 01: 99-101

[2] LI Tao. An Analysis of the Status of Community Taijiquan in Taiyuan [J]. Journal of Physical Education Institute of Shanxi Normal University, 2008, S1:49-51.

[3] Liu Chuanqin, Zhu Chunwang, Shen Yong. A Survey and Research on Community Taijiquan in Mudanjiang [J]. Adult Education, 2009, 09: 68-69. 
[4] Tong Maohua. An Analysis of Development Status of Community Taijiquan in Jiaozuo [J]. Sports World Scholarly, 2009, 10: 119-120.

[5] Xie Xiaoju. An Investigation and Research of Community Taijiquan [J]. Wushu Science, 2006, 10: 30-31. 